\title{
ON COARSE-GRAINED ENTROPY AND MIXING IN STATISTICAL MECHANICS
}

\author{
C.G. Chakrabarti \\ Department of Applied Mathematics \\ University of Calcutta \\ Calcutta - 700009, INDIA
}

(Recelved January 7, 1985 and in revised form October 22, 1986)

ABSTRACT. The object of the paper if the study the roles of non-equilibrium entropy and mixing of phases in the statistical characterization of the coarse-grained interpretation of the irreversible approach to statistical equilibrium of an isolated system.

KEY WORDS AND PHRASES. Probability space, $\sigma$-algebra, observational states, sufficlent partition, Coarse-grained distribution, Ergodicty, and Weak-mixing. 1980 AMS SUBJECT CLASSIFICATION CODE. $82 A 05$.

\section{INTRODUCTION.}

The process of coarse-graining which is equivalent to the statistical averaging of the micro-states over the various phase-cells plays a significant role in the study of the macroscopic property of irreversibility from the reversibility of dynamical equations of motion. The coarse-graining cannot be done arbitrarily and Giggs entropy based on an arbitrary coarse-grained distribution does not always ensure the relaxation to statistical equilibrium. The object of the present paper is to introduce a non-equilibrium entropy after Goldstein and Penrose [1] and to study the importance of ergodicity and mixing in the statistical characterization of the irreversible approach to statistical equilibrium of a classical isolated system.

2. DYNAMICAL SYSTEM AND OBSERVATIONAL STATES.

Let us consider a classical dynamical system whose dynamical state if given by $\left(\Omega, \mathrm{T}_{t}\right.$ ) where $\Omega$ is the phase-space consisting of all possible phase-points and $\left\{\mathrm{T}_{t}\right\}$ is the family of time-evolution transformations (automorphisms) defined for all real $t$ generated by the dynamical equation of motion in phasespace $\Omega$. Let $m$ be the invariant Liouville's measure of phase-space and let $\nu$ be any other measure absolutely continuous to $m$. The probability density of microstates $\rho(\omega)$ is defined by a normalized density given by the Radon-Nikodym derivative

$$
\rho(w)=\frac{d v}{d n}(w)
$$


The time-evolution of the measure $\nu$ consists of the family of measures $\left[\nu_{t}\right\}$ defined by [1]

$$
\nu_{t}\left(T_{t} \omega\right)=v(\omega)
$$

This implies that for any set $A \in \Omega$

$$
v_{t}(A)=\left(T_{-t} A\right) \text {. }
$$

The statistical structure (or model) of the system by the probabilityspace ( $\Omega, A,\left\{\nu_{t}\right\}$ ) where $A$ is the $\sigma$-algebra of subsets of $\Omega$ including itself, and, $\left\{\nu_{t}\right\}$ is the family of probability measures on $A$.

To determine the observational states of the system at the initial time $t=0$ we divide the time of observation into a countably infinite number of intervals of equal length. Considering the length of each interval as the unit of the measurement of time, the evolution of a subset $A$ in the course of $t$ ime is given by the series $T_{t} A$ $(t=\ldots-2,-1,0,1,2 \ldots)$. Let us define a partition $P$ of phase-space into sets of points that are indistinguishable by an observation made at $t$ ime $t=0$. Two phase points $\omega_{1}$ and $\omega_{2}$ are then observationally equivalent if and on $1 y$ if the time translates $T_{t} \omega_{1}$ and $T_{t} w_{2}$ which $11 e$, for every non-negative integer $t$, in the same set from the partition $P$. In other words, $\omega_{1}$ and $\omega_{2}$ must lie in the same set from the partition $T_{t} P$. Let us define the $\sigma$-algebra

$$
\dot{a}=\stackrel{\mathbb{V}}{=}_{0}^{\infty} T_{-t} P
$$

as the smallest $\sigma$-algebra which contains all the partitions $P, T_{-1} P_{,} T_{-2} P, \ldots$ Thus every set in is is the image under of some set in $a$; that is, to say, the o-algebra $T$ consists of image under $T_{t}$ of all sets belonging to and Includes (among others) all the sets of $a$ itself [1]:

$$
T_{t} a \supset a
$$

The condition (2.5) is the condition of loss of observational information and represents the asymmetry between past and future [1].

3. NON-EQUILIBRIUM ENTROPY: IRREVERSIBILITY AND MIXING.

The entropy (fine-grained) of the classical dynamical system is defined by the functional

$$
\begin{aligned}
& S\left(\nu_{t}, A\right)=-K \int \rho_{t}(\omega) \log \rho_{t}(\omega) \operatorname{dm}(\omega) \\
& =-k \int \log \left(d \nu_{t} / d m\right) d \nu_{t}(\omega) .
\end{aligned}
$$

The entropy $S\left(\nu_{t}, A\right)$ defined over the fine grained-density $\rho_{t}(\omega)$ contains full infromation about the system. For observation behavior of the system such detailed information or description about the system is not necessary. For this a coarsegraining of microstates is necessary [2].

Let us consider the coarse-grained density $\bar{\rho}_{t}$ as the conditional expectation of $\rho_{t}(\omega)$ with respect to the $\sigma$-algebra $a$ (which is the consitional expectation for the 
measure $\mathrm{m} / \mathrm{m}(\mathrm{s} z))[1]$.

$$
\bar{\rho}_{t}=E(\rho(\omega) \mid a)
$$

The entropy for the non-equilibrium state is then defined by the coarse-grained entropy [1]

$$
\tilde{s}\left(\nu_{t}, a\right)=-K \int \bar{\rho}_{t} \log \bar{\rho}_{t} \mathrm{dm}(\omega)
$$

Since the measure $m$ used in defining the entropy is invariant, we have,

$$
\tilde{s}\left(\nu_{t}, a\right)=\tilde{s}\left(\nu, T_{-t} a\right)
$$

From (2.5), we have

$$
\mathrm{T}_{-t} a \subset a,(t>0)
$$

As a consequence of $(3.5)$, it is easy to prove that

$$
\tilde{s}\left(\nu, T_{-t} a\right)>\tilde{s}(\nu, a)
$$

or by $(3.4)$, we have

$$
\tilde{s}\left(\nu_{t}, a\right)>\tilde{s}(\nu, a), \quad(t>0)
$$

which proves the non-decreasing property of the entropy $\tilde{s}\left(\nu_{t}, a\right)$ with time; that is, the H-theorem.

The equality in (3.7) corresponds to the stationary state of statistical equilibrium of the system at the initial time $t=0$. Mathematically this holds for the measure-preserving automorphism $\mathrm{T}_{t}$ :

$$
v_{t}=v
$$

or

$$
v_{-t}=v_{0}
$$

Also the equality in (3.6), which is a consequence of the relation ( 3.5$)$, holds for the invariance relation:

$$
\mathrm{T}_{-t} a=a
$$

Thus the inequality in (3.7) for statistical equilibrium at the initial time $t=0$ corresponds to the invariance of the $\sigma$-algebra a under measure-preserving automorphism $T_{t}$ that is, to the condition of ergodicity of the system. The equality has also an important statistical significance. This, in fact corresponds to the sufficiency of the $\sigma$-algebra $a$ or to the sufficient partitioning of microstates (or phase-space) into equivalent class of macrostates of the system [3.4]. The $\sigma-$ algebra $a$ is sufficient for the famlly of probability measures $\left\{\nu_{t}\right\}$ if the conditional expectation of any dynamical variable, say Hamiltonian $X(\omega)$ given the $\sigma-a l g e b r a a$, that 1s, If $E\{X(\omega) \mid a\}$ is the same for all $\nu_{t} \in\left\{\nu_{t}\right\}[3]$. The sufficiency of the $\sigma-$ algebra a implies the time-variance of the conditional probability density $E\{\rho(\omega) \mid a\}$ which by definition is our coarse-grained density under 
consideration. The different non-null atoms of the sufficient $\sigma-a l g e b r a$ a represent the different macrostates of statistical equilibria of the system at the initial time $t=0$. This is a significant result. In an earlier paper [4], we have in fact shown that the sufficiency of the $\sigma$-algebra a for statistical equilibrium results from the ergodicity of the system.

The non-decreasing property of the entropy $\tilde{s}\left(\nu_{t}, a\right)$ is, however, not sufficient to ensure the relaxation to equilibrium over the phase-space (energy-shel1) $\Omega$. For this a more broad assumption, namely the assumptionof mixing of phases is necessary. That the coarse-grained distribution generated by the $\sigma$-algebra $a$

corresponds to the process of mixing results from the relation: $T_{t} a \supset a$. For measure-preserving automorphism $T_{t}$, the sequence $\left\{T_{t} a\right\}$ forms a monotonically increasing sequence of $\sigma$-algebra and let $a_{\infty}$ where

$$
a_{\infty}=\stackrel{v}{v}=0_{t} T_{t}
$$

be its limit in the sense that $\mathrm{T}_{t} a \uparrow a_{\infty}$. Note the $a_{\infty}$ being the smallest J-algebra which includes all sets belonging to $T_{t} l(t=0,1,2 \ldots)$ is, therefore, equal to the $\sigma-$ algebra $\left\{\phi, S_{2}\right\}$, consisting of the null-set $\phi$ and the phase-space (ergodic set) $\Omega$ Then by Doob's convergence theorem [5]

$$
\lim _{t \rightarrow \infty} \nu\left\{A \mid T_{t} a\right\}=\nu\left\{A \mid a_{\infty}\right\}
$$

which is the condition of weak-mixing or relaxation to statistical equilibrium [6]. To express it in a more familiar form we note that the $\sigma$-algebra $a_{\infty}=\{\phi, \Omega\}$ comprises of all sets of measure 0 and $\mathrm{m}(\Omega)$. The mixing condition $(3.11)$, then implies the convergence of the coarse-grainded density $\bar{\rho}_{t}$ to the statistical equilibrium (microcanonical) density $1 / \mathrm{m}(\Omega)$ :

or

$$
\begin{aligned}
& \lim _{t \rightarrow \infty} \bar{\rho}_{t}=1 / \mathrm{m}(\Omega) \\
& \lim _{t \rightarrow \infty} \tilde{S}\left(\nu_{t}, a\right)=\mathrm{K} \log \mathrm{m}(\Omega)
\end{aligned}
$$

where the r.h.s is the thermodynamic equilibrium entropy. Thus, while the ergodicity corresponds to the states of statistical equilibria over the various phase-cells (nonnullatoms of $a$ ) at the initial time $t=0$, the mixing of phases ensures the $11 \mathrm{mit}$ ing case of relaxation of the system to statistical equilibrium over the whole of phasespace $\Omega$ of the system.

4. CONCLUSIONS.

The paper aims to stress the importance of the properties of ergodicity and mixing in the coarse-grained interpretation of the irreversible approach to statistical equilibrium. The analysis is based on a measure of entropy defined for the non-equilibrium states of an isolated system. The invariance of the $\sigma$-algebra a under measure-preserving automorphism $T_{t}$ corresponds to the statistical equilibria over the various phase-cells (including the whole phase-space $\Omega$ also) at the initial time $t=0$. The sufficiency-a reduction principle of statistics, plays a significant role in the statistical characterization of statistical equilibria at the initial time. In the case of initial non-equilibrium distribution, it is, however, the assumption of phase-mixing which ensures the relaxation to statistical equilibrium over the whole of phase-space [4]. 


\section{REFERENCES}

1. Goldstein, S. and Penrose, C. J. Stat. Phys. 24(1981), 325.

2. FARQUHAR, F. Ergodic Theory in Statistical Mechanics, Interscience Publ., New York, 1964.

3. KULLBACK, S. Information Theory and Statistics, Wiley and Sons, 1959.

4. CHAKRABART I, C.G. J. Math. and Phys. Sc1. 9(1975), 381. Report Math. Phys.

16(1969), 143. Z. Phys. B51(1983), 265. Z. Phys. B61(1985), 353.

5. BILLINGSLEY, P. Ergodic Theory and Information, Wiley and Sonc, New York,1965.

6. HOBSON, A. Concepts of Statistical Mechanics, Gordon and Breach, San Francisco, 1970. 


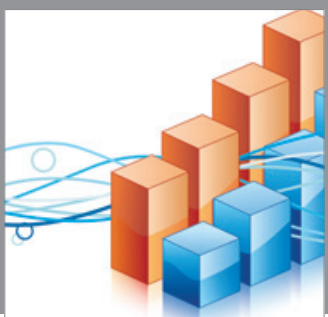

Advances in

Operations Research

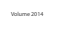

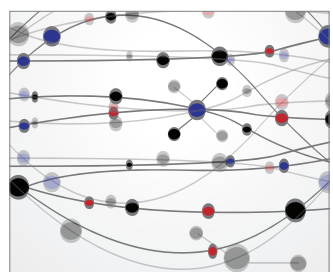

\section{The Scientific} World Journal
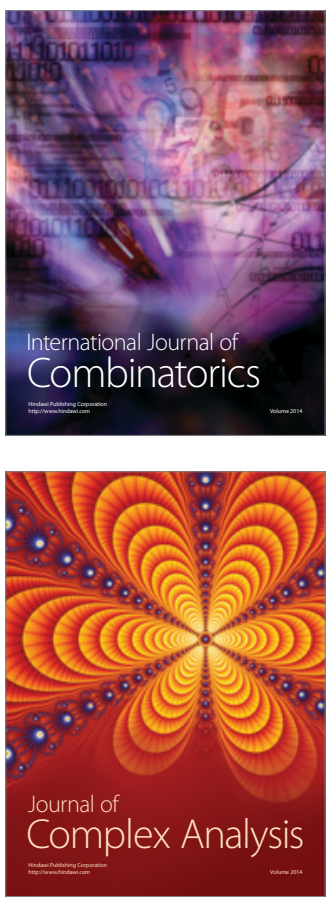

International Journal of

Mathematics and

Mathematical

Sciences
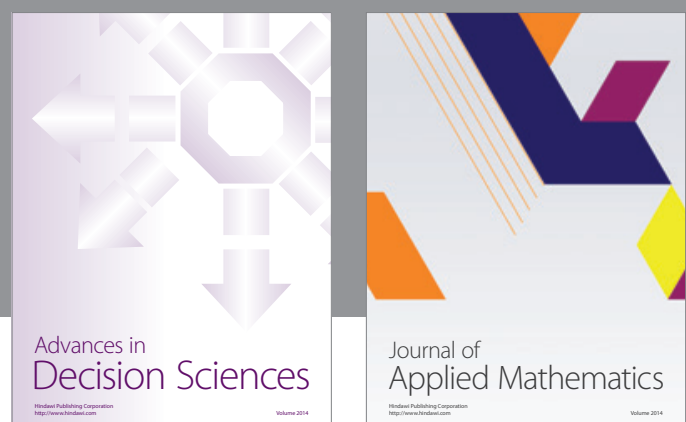

Journal of

Applied Mathematics
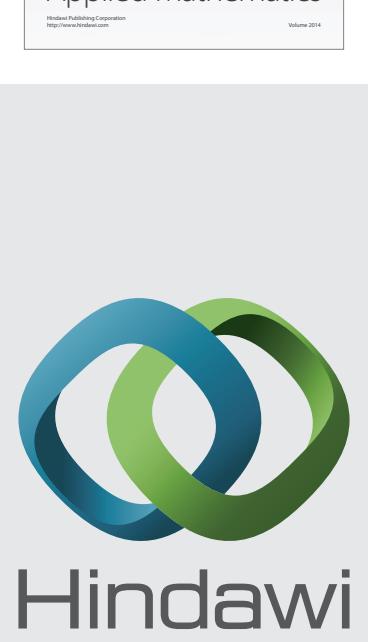

Submit your manuscripts at http://www.hindawi.com
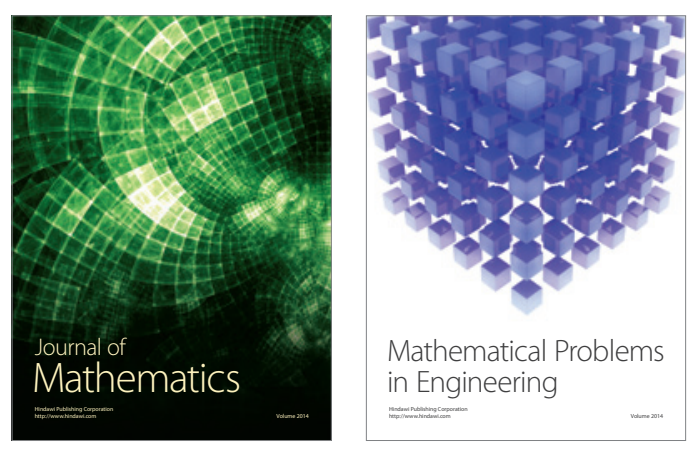

Mathematical Problems in Engineering
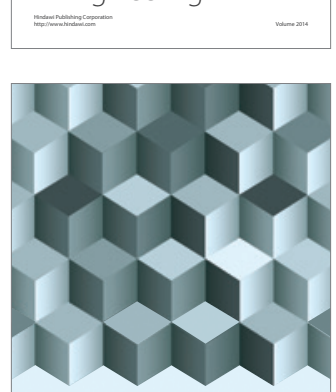

Journal of

Function Spaces
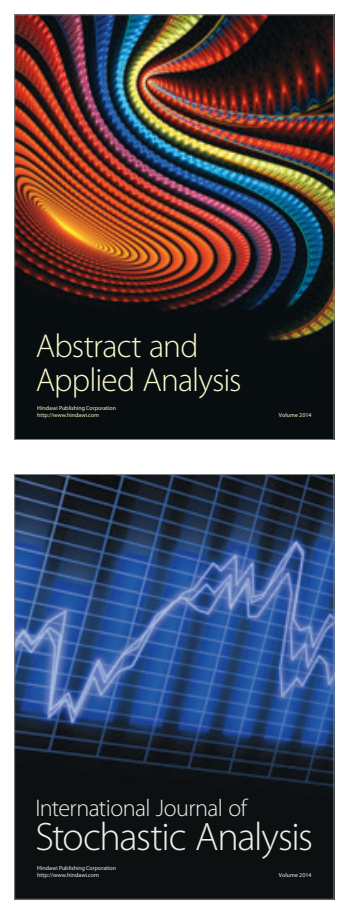

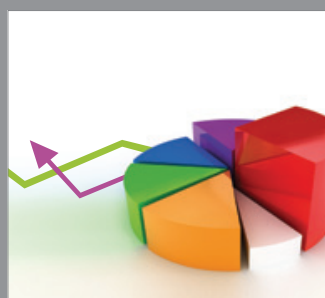

ournal of

Probability and Statistics

Promensencen
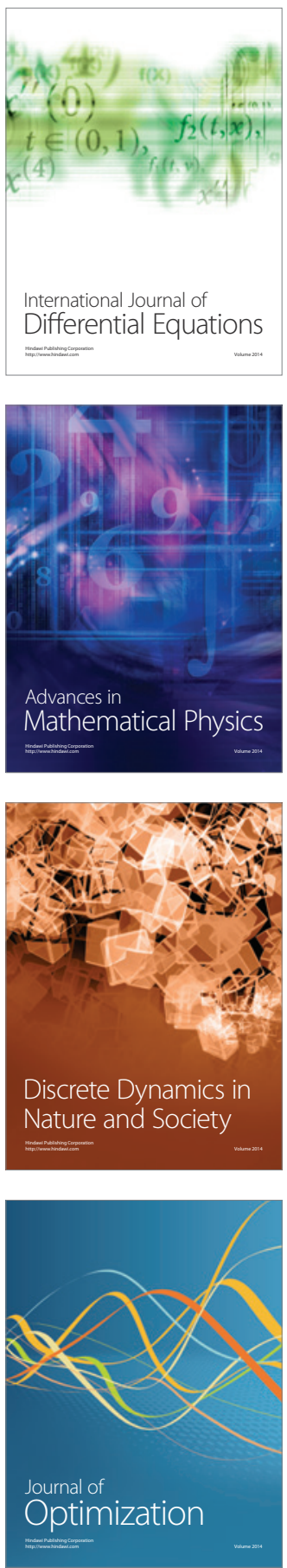